\title{
Numerical Study on the Transient Preheating Process of a Regenerative Oxidation Bed
}

\author{
Yongqi Liu*, Jinhui Han, Yanyan You and Zhenqiang Gao \\ School of Transportation and Vehicle Engineering, Shandong University of Technology, Zibo 255049, China
}

\begin{abstract}
The transient preheating process of a regenerative oxidation bed placed in a coal mine methane thermal reverse-flow reactor is numerically investigated in this paper. The regenerative oxidation bed is heated by the burned gas which is generated from a burner and is distributed through manifolds, which is modeled in a three-dimensional, unsteady state and laminar flow system, and is assumed as a semitransparent porous media. Non-local thermal equilibrium between gas and solid is accounted for introducing separate energy equations for two phases in porous media, and the finite volume method is used to solve the radiative transfer equation of the solid phase to calculate the local radiation source term. The calculating results indicate that the regenerative oxidation bed is preheated mainly by the hot gas passing through the manifold, and increasing the inlet mass flow could shorten the time of preheating, specific heat and porosity have a great influence on the heat storage capacity of the regenerative oxidation bed, and the contribution of the solid phase radiation to heat transfer can not be ignored, the temperature is highest in the middle of the regenerative oxidation bed, and it decreases gradually along the direction of height of the regenerative oxidation bed. All these work can help to understand the transient preheating process of a regenerative oxidation bed and the heat transfer phenomena and mechanism during the process.
\end{abstract}

Keywords: Preheating, regenerative oxidation bed, heat transfer, porous media, manifold.

\section{INTRODUCTION}

Methane vented from coal mine exhaust shafts constitutes an unused source of energy and a potent atmospheric greenhouse gas (GHG). Technologies that can reduce ventilation air methane (VAM) emissions while harnessing methane's energy offer significant benefits to the world community. The thermal flow-reversal reactor (TFRR) and the catalytic flow-reversal reactor (CFRR) are both candidates for utilizing the low methane concentrations contained in VAM streams [1-3]. TFRR has been commercially used in Australia and China, but no reports of commercial applications of CFRR are found [4-6].

The regenerative oxidation bed, consisting of a number of honeycomb ceramic, is the site for methane oxidation in TFRR [7]. To start the operation, the middle of the regenerative oxidation bed must be preheated to the temperature required to initiate methane oxidation or hotter by the electric heating elements embedded in it or the burned gas generated from a burner placed outside. For the second case, the burned gas coming from the burner is transported to the middle of the regenerative oxidation bed, and is distributed through manifold. During the preheating process, the temperature and heat storage capacity of the regenerative oxidation bed increases gradually due to the heat transfer between the hot gas and the bed. The mechanism of the heat transfer is very complicated, including radiation, conduction and convection [8-11].

*Address correspondence to this author at the School of Transportation and Vehicle Engineering, Shandong University of Technology, Zibo 255000, China; Tel: +86-533-2782616 (Office), +86-13969353396 (Mobile); Fax: +86-533-2786837; E-mail: liuyq65@163.com
In recent years, there were numerous investigations on heat transfer in a porous media [12-14]. C. Ben Kheder, B. Cherif and M.S. Sifaoui studied numerically the transient heat transfer in semitransparent porous medium using a fully implicit time-marching algorithm to solve the nonlinear coupled energy equations for gas and porous medium, and reported that the Reynolds number, optical characteristic and conduction-radiation parameter have obvious effects on the temperatures.

The main purpose of this study is to numerically investigate the transient preheating process of the regenerative oxidation bed heated by the burned gas, and analyze the effects of some important parameters on the heat storage capacity in order to better understand the heat transfer phenomena and mechanism during the preheating process.

\section{PHYSICAL MODEL}

The coal mine VAM TFRR which was made and tested at Shan Dong University of Technology is used in this study, as shown in Fig. (1). It is a simple apparatus that consists of a regenerative oxidation bed, a heating and starting system, an upper header and a lower header, four control valves and ducts. The regenerative oxidation bed, consisting of a number of honeycomb ceramic, is the main part of the TFRR, where the methane is oxidized and the energy is stored. It is a vertical structure $(2100 \mathrm{~mm} \times 1350 \mathrm{~mm} \times 2400 \mathrm{~mm})$, in which gas flows along the vertical direction, as shown in Fig. (1). In the middle of the TFRR is the hot gas distribution system of the heating and starting system, through which the hot gas is distributed and the oxidation bed is heated uniformly within the starting stage. The upper header and the lower header are used to guide the feed into the oxidation 


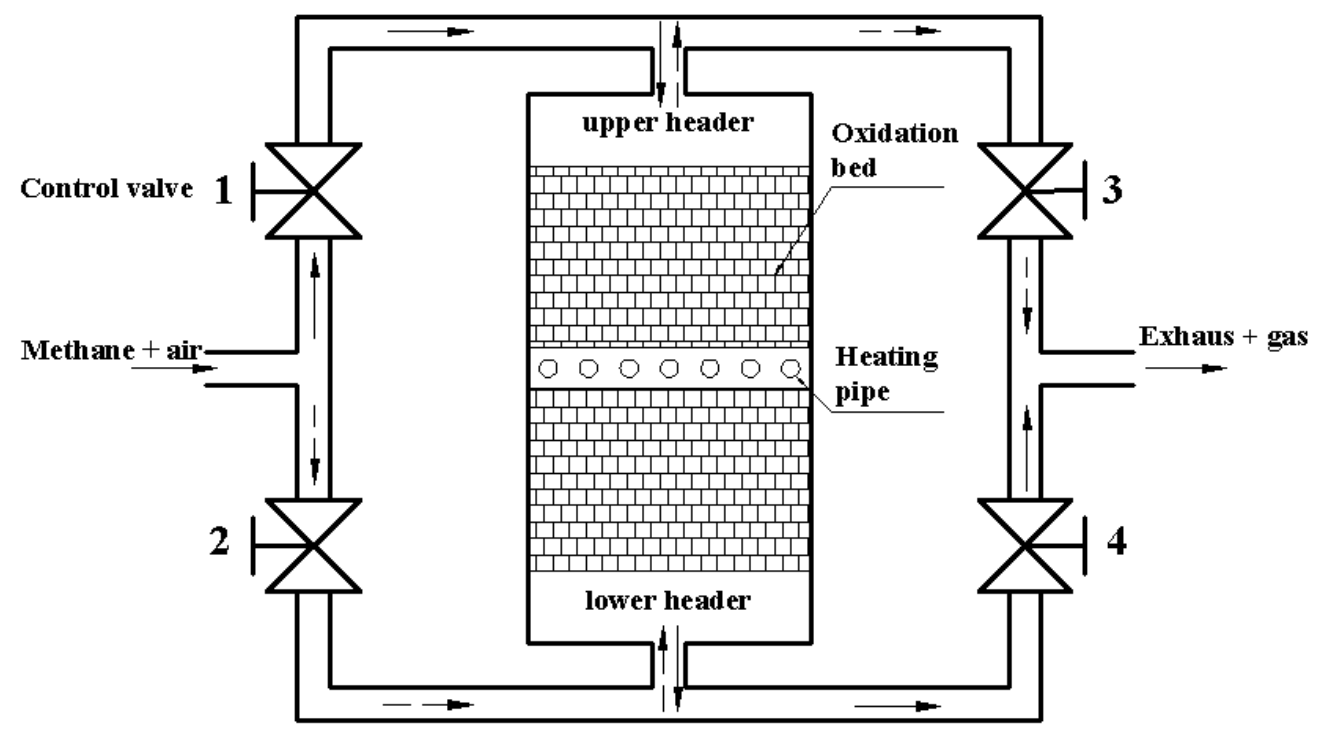

Fig. (1). The schematic of the coal mine VAM TFRR.

bed uniformly. Four control valves are used to control the direction of VAM flowing in the regenerative oxidation bed.

The operating process employs the principle of regenerative heat exchange between a gas (ventilation air) and a solid (bed of honeycomb ceramic). One cycle of the process is comprised of two flow reversals, so each flow reversal is a half-cycle. Referring to Fig. (1), assume that during the first half-cycle both the control valves 1 and 4 are open while the control valves 2 and 3 are closed. Thus, the flow through the reactor takes place from bottom to top. After a time interval, the reactor reverses flow direction by closing the control valves 1 and 4 and opening the control valves 2 and 3. Flow then takes place from top to bottom.

To start the operation, the burned hot gas coming from the heating and starting system preheat the middle of the regenerative oxidation bed to the temperature required to initiate methane oxidation (above $900^{\circ} \mathrm{C}$ ) or hotter. This preheating process takes about experiences dozens of hours. After completion of the preheating process, VAM at ambient temperature enters and flows through the oxidation bed in one direction, and its temperature increases until oxidation of the methane takes place near the center of the oxidation bed. The hot oxidate continue through the oxidation bed, transferring heat to the far side of the oxidation bed. When the far side of the oxidation bed is sufficiently hot, the reactor automatically reverses the direction of VAM. The VAM now enters the far (hot) side of the oxidation bed, where it is heated gradually to auto-oxidation temperatures near the center of the oxidation bed and then oxidizes.

Fig. (2) shows the schematic of the heating and starting system, which consists of two parts, one is the hot gas production and adjustment system, the other is the hot gas distribution system. The burned gas coming from the burner and the air coming from the blower are mixed in hot gas mixing chamber firstly. In the hot gas mixing chamber, a thermocouple is used to measure the temperature of the mixing hot gas. And in order to get some certain temperature of the mixing hot gas, a valve is needed to control the mass flow of the air. Fig. (3) shows the sectional view of the middle part of the regenerative oxidation bed. The manifolds placed in the center of the oxidation bed have many holes through which the hot gas is distributed uniformly. In order to reduce workload, only one manifold is chosen to be studied in this paper, as shown in Fig. (4).

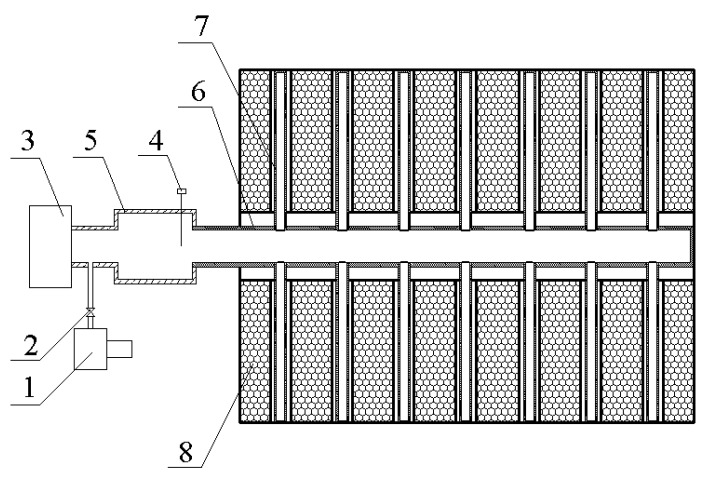

Fig. (2). The schematic diagram of the system of heating and starting (1-blower; 2-valve; 3-burner; 4-thermocouple; 5-hot gas mixing chamber; 6- main pipe; 7-manifold; 8- honeycomb ceramic).

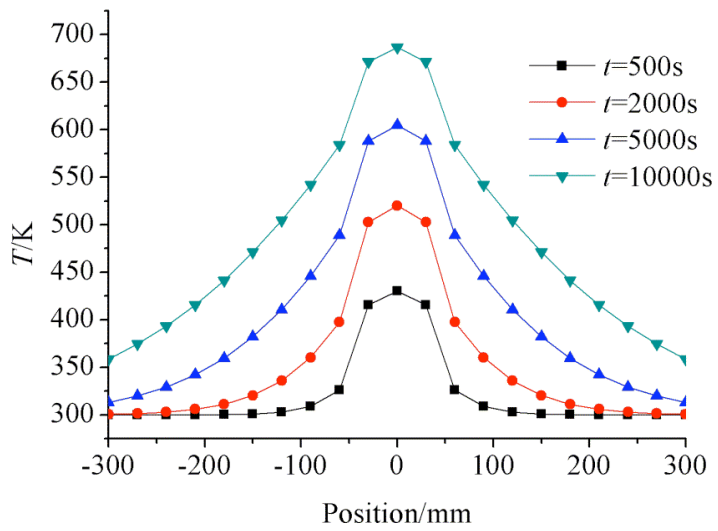

Fig. (3). The schematic diagram of the regenerative oxidation bed. 


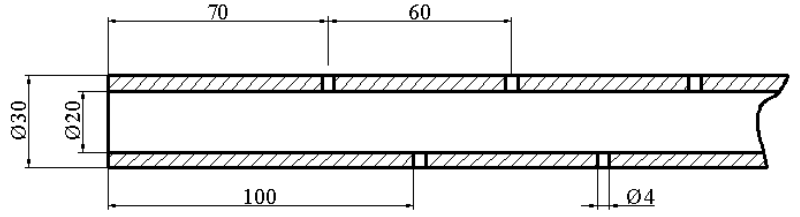

Fig. (4). The sketch of the manifold.

\section{GOVERNING EQUATION}

For the mathematical analysis, the following basic assumptions are made: (a) the honeycomb ceramic is assumed as porous media; (b) the mass and heat fluxes of the hot air through the holes are uniform; (c) the hot air is compressible and its thermophysical properties are designed as piecewiselinear of temperature, and is listed in Table 1; (d) the radiation of the hot air is negligible; (e) the gas flow in porous media is assumed to be laminar; (f) the thermophysical properties of all porous media are taken to be constants.

Table 1. The Thermophysical Properties of the Hot Gas

\begin{tabular}{|c|c|c|c|c|}
\hline$\frac{T}{K}$ & $\frac{\rho}{\mathrm{kg} / \mathrm{m}^{3}}$ & $\frac{c_{p}}{\mathrm{~kJ} /(\mathrm{kg} \cdot \mathrm{k})}$ & $\frac{\lambda \times 10^{2}}{W /(\mathrm{m} \cdot \mathrm{K})}$ & $\frac{\mu \times 10^{6}}{\mathrm{~kg} /(\mathrm{m} \cdot \mathrm{s})}$ \\
\hline \hline 273 & 1.293 & 1.005 & 2.44 & 17.2 \\
\hline 473 & 0.746 & 1.026 & 3.93 & 26.0 \\
\hline 673 & 0.524 & 1.068 & 5.21 & 33.0 \\
\hline 873 & 0.404 & 1.114 & 6.22 & 39.1 \\
\hline 1073 & 0.329 & 1.156 & 7.18 & 44.3 \\
\hline 1273 & 0.277 & 1.185 & 8.07 & 49.0 \\
\hline
\end{tabular}

Based on these assumptions, the governing conservation equations used in this simulation calculation to solve the problems are given as follows.

For a three-dimensional, unsteady state and laminar flow system, the continuity equation can be written as

$$
\frac{\partial \rho_{g}}{\partial t}+\nabla\left(\rho_{g} \vec{v}\right)=0
$$

where $\rho_{\mathrm{g}}$ is the hot gas density, $\mathrm{kg} \cdot \mathrm{m}^{-3} ; v$ is the hot gas velocity, $\mathrm{m} \cdot \mathrm{s}^{-1}$.

The momentum conservation equation of the gas in porous media can be described by the Darcy law

$$
\nabla p=-\frac{\mu}{\alpha} \vec{v}
$$

in which, $\alpha$ is permeability of porous media; $\mu$ is the hot gas viscosity, pa·s.

The temperature between gas and solid is different during the preheating process, so separate energy equations for the two phases are introduced [15, 16]. Solid phase energy equation is expressed as

$$
(1-\varphi)(\rho c)_{s} \frac{\partial T_{s}}{\partial t}=(1-\varphi) \nabla \cdot\left(\lambda_{s} \nabla T_{s}\right)+h\left(T_{g}-T_{s}\right)-\nabla \cdot \vec{q}
$$

in which, $T$ is temperature, $\mathrm{K} ; c$ is specific heat, $\mathrm{J} \cdot \mathrm{kg}^{-1} \cdot \mathrm{K}^{-1} ; h$ is volumetric the heat transfer coefficient, $\mathrm{W} \cdot \mathrm{m}^{-3} \cdot \mathrm{K}^{-1} ; \phi$ is porosity; $q$ is radiative heat flux, $\mathrm{W} \cdot \mathrm{m}^{-2} ; \lambda$ is thermal conductivity of the medium, $\mathrm{W} \cdot \mathrm{m}^{-1} \cdot \mathrm{K}^{-1}$. The subscripts " $g$ " and " $s$ " stand for gas and solid phase, respectively.

Gas phase energy equation is expressed as,

$$
\varphi\left(\rho c_{p}\right)_{g} \frac{\partial T_{g}}{\partial t}+\left(\rho c_{p}\right)_{g} V \cdot \nabla T_{g}=\varphi \nabla \cdot\left(\lambda_{g} \nabla T_{g}\right)+h\left(T_{g}+T_{s}\right)
$$

The porous media in this study is considered as a gray medium. The heat source term due to radiation that appears in equation ( 3 ) is calculated by,

$$
\nabla \cdot \vec{q}=\kappa\left\{4 \pi I_{b}[T(\vec{r})]-\int I(\vec{r}, \vec{s}) d \Omega\right\}
$$

where $\kappa$ is the absorption coefficient, $\mathrm{m}^{-1} ; \Omega$ is solid angle, $\mathrm{sr} ; I$ is radiation intensity, $\mathrm{W} \cdot \mathrm{m}^{-2}$, calculated by

$$
\begin{aligned}
& (\vec{s} \cdot \nabla) I(\vec{r}, \vec{s})=-\beta I(\vec{r}, \vec{s})+\kappa I_{b}(\vec{r}) \\
& +\frac{\sigma_{s}}{4 \pi} \int_{4 \pi} I(\vec{r}, \vec{s}) \phi\left(s^{r^{\prime}} \rightarrow \vec{s}\right) d \Omega
\end{aligned}
$$

where $\beta$ is the extinction coefficient, $\mathrm{m}^{-1} ; \sigma_{s}$ is the scattering coefficient, $\mathrm{m}^{-1}$.

\section{NUMERICAL METHOD}

The three-dimensional coupled heat transfer problem is solved using the software FLUENT6.2. Laminar flow is assumed for the gas flow in porous media and the $K-\varepsilon$ turbulent model is used in the numerical calculations for the turbulent flow in the manifold. Pressure and velocity are coupled by the SIMPLE algorithm. The discrete ordinate method (DOM) is used to solve the radiative transfer equation $[17,18]$.

Because the hot air is compressible, so the mass flow boundary is used for the inlet boundary condition of the manifold.

For the hot air

$q_{0}=q_{\text {in }}, T_{0}=T_{\text {in }}$

$T_{\text {in }}, q_{\text {in }}$ are initial temperature and mass flow of the hot air. Initially, the porous media is at room temperature throughout

At the inner wall of the manifold, coupled thermal conditions is chosen, where

$T_{g}=T_{w}$

$T_{\mathrm{g}}$ is the temperature of the hot air nearby the inner wall of the manifold, $T_{\mathrm{w}}$ is the temperature of the inner wall of the manifold. 
At the regenerative oxidation bed wall, the usual no-slip and impenetrability condition is applied. These surfaces are assumed to be gray, emitting and reflecting diffusely. Therefore, the boundary intensity for outgoing direction is

$$
I(\vec{r})=\varepsilon I_{b}(\vec{r})+\frac{1-\varepsilon}{\pi} \int_{\vec{s} \cdot n \leq 0} I(\vec{r}, \vec{s})|\vec{s} \cdot n| d \Omega
$$

where $n$ and $\varepsilon$ are unit vector normal to the surface and emissivity, respectively.

The top and bottom of the regenerative oxidation bed are defined as outlet, where pressure outlet boundary is introduced due to the reverse flow of the hot gas. And the radiation transfer between the porous media and environment is considered.

$$
(1-\varphi) \lambda_{s} \frac{\partial T_{s}}{\partial z}=-\varepsilon \sigma\left(T_{s}^{4}-T_{0}^{4}\right), I=\frac{\sigma T_{s}^{4}}{\pi}
$$

\section{RESUILTS AND DISCUSSION}

Because the regenerative oxidation bed is structure symmetrical along the vertical direction, as shown in Fig. (1). And the hot gas is distributed through the hot gas distribution system located in the middle of the regenerative oxidation bed. So the top of the regenerative oxidation bed is chosen in the following analysis at the heating and starting stage. Fig. (5) shows the temperature difference between gas and solid phase in the regenerative oxidation bed at the conditions of $T_{\text {in }}=1200 \mathrm{~K}, q_{\text {in }}=0.00155 \mathrm{~kg} \cdot \mathrm{s}^{-1}$. In Fig. (5), the $\mathrm{x}$-coordinate represents the position in the regenerative oxidation bed along the vertical direction and the middle of the regenerative oxidation bed is defined at $x=0$. The $y-$ coordinate represents the change of temperature. It can be seen that the temperature of gas is higher than the solid's. Along the vertical direction, the difference of temperature becomes smaller gradually, which means the heat transfer between the two phases is more completely.

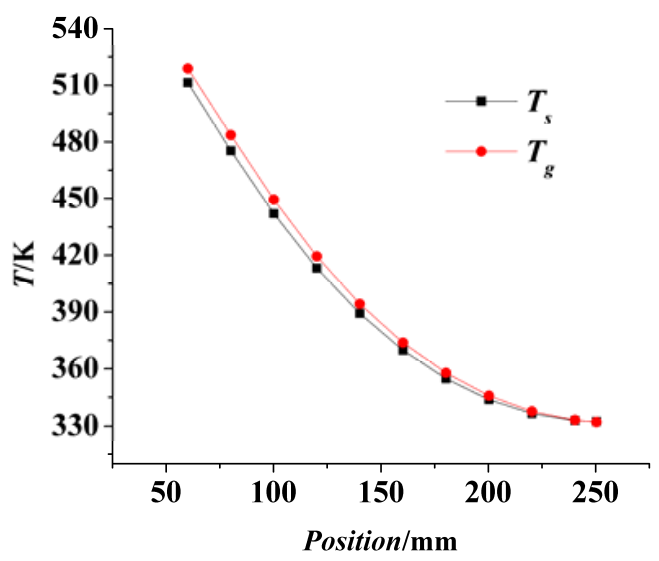

Fig. (5). The temperature of gas and solid phase.

\subsection{Ways of Preheating of the Porous Media}

Fig. (6) shows the changes of different ways of preheating during the preheating process. It can be seen that the porous media can be preheated directly by the hot gas passing through the manifold and the heat transfer between the outer wall of the manifold and the porous media. Besides, Fig. (6) shows that the heat mass flow passing through the manifold is less than $40 \%$ at the beginning of the preheating process, but it increases with the increase in heating time. Within the first $100 \mathrm{~s}$, because the temperature of the outer wall of the manifold is less than that of hot gas outside of the manifold, the heat flow between the outer wall of the manifold and the porous media keeps zero. The heat transfer between the outer wall of the manifold and the porous media reaches to the biggest value at the time of 1000 s, and then it turns down and becomes smaller and smaller with the increase of the preheating time.

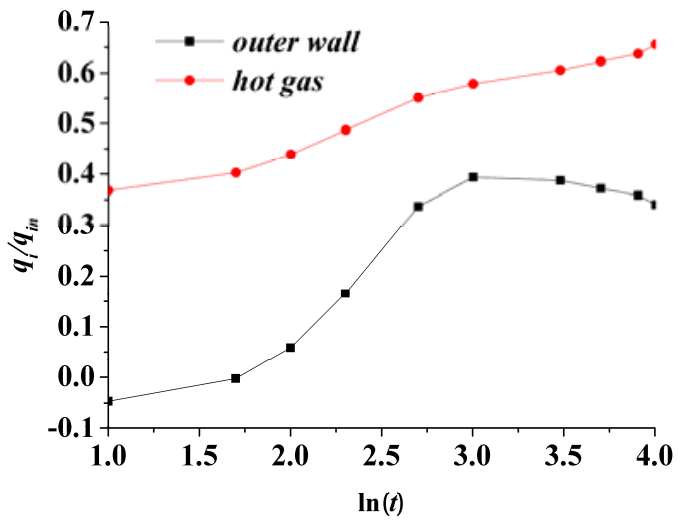

Fig. (6). The changes of different ways of preheating during the preheating process.

The transient temperature distribution of porous media for various inlet mass flows is shown in Fig. (7). It is seen that the temperature of the regenerative oxidation bed increases with the increase in inlet mass flow during preheating process. This phenomenon can be understood as that the greater the inlet mass flow is, the more heat energy is transported to the porous media within one unit time.

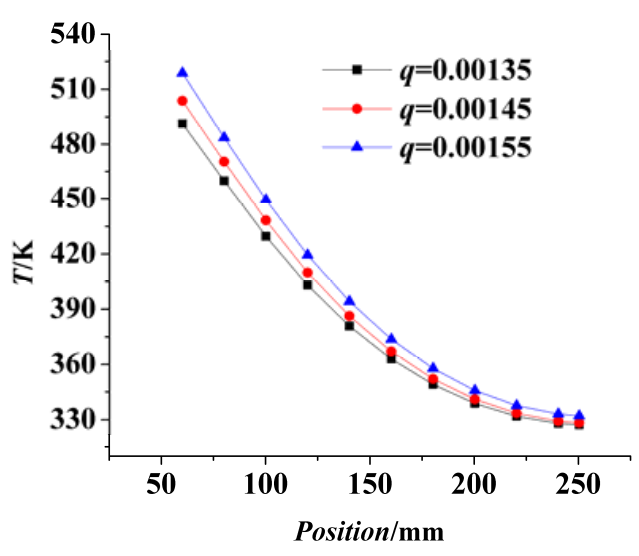

Fig. (7). The temperature distribution for different inlet mass flow.

\subsection{Influence of Specific Heat of the Porous Media}

Fig. (8) illustrates the effect of specific heat of the porous media on the temperature of the outlet at different time. The conditions are: $T_{\text {in }}=1200 \mathrm{~K}, q_{\text {in }}=0.00155 \mathrm{~kg} \cdot \mathrm{s}^{-1}$. From Fig. (8), 
it can be seen that the temperature of the outlet decreases significantly with the increase in the specific heat after the bed is preheated for a long time. At the time of $3000 \mathrm{~s}$, the temperature difference is even over $50 \mathrm{~K}$.

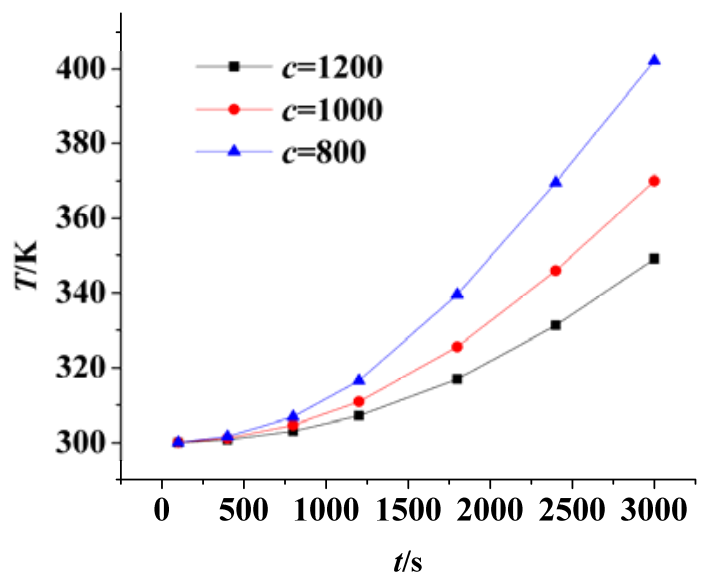

Fig. (8). The outlet temperature of porous media for different specific heat.

This fact can be explained that specific heat is an important parameter affecting the heat storage capacity of porous media. The larger the specific heat is chosen, the greater the heat storage capacity will be gained. That is to say when the inlet mass flow fixed, more heat energy would be stored by the regenerative oxidation bed with high specific heat.

\subsection{Influence of Porosity}

At the conditions of $T_{\text {in }}=1200 \mathrm{~K}, \quad q_{\text {in }}=0.00155 \mathrm{~kg} \cdot \mathrm{s}^{-1}$, $t=2000 \mathrm{~s}$, the effects the porosity of the porous media on the heat transfer are shown in Fig. (9). It can be seen that the trends of temperature distribution for different porosity along the vertical direction of the regenerative oxidation bed are similar. But the higher porosity, the higher temperature profile for the regenerative oxidation bed.

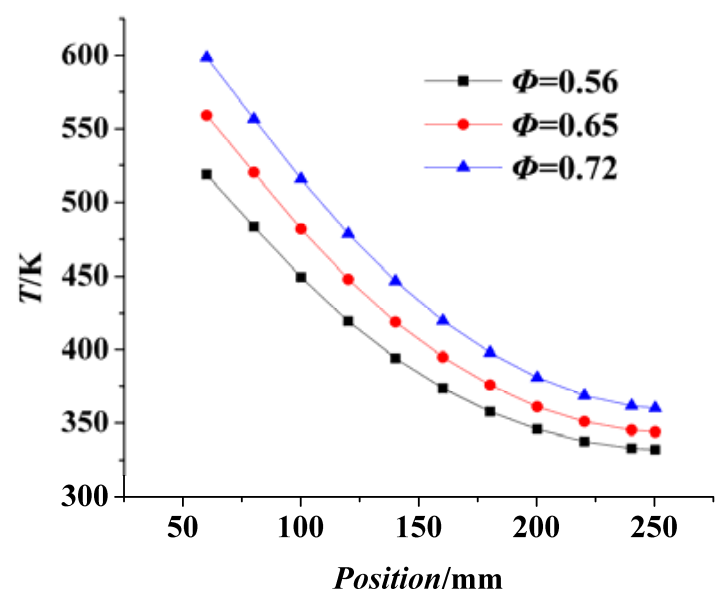

Fig. (9). The temperature distribution for different porosity.

The reasons are explained as follows. The higher porosity is, the smaller density for certain volumetric porous media is. So the heat storage capacity of porous media would decreases with the decrease in density, and the temperature will rise quickly along the vertical direction in the regenerative oxidation bed accordingly, resulting in the temperature of the outlet increase and more heat energy loss.

\subsection{Influence of Radiation Heat Transfer}

Fig. (10) shows the difference of enthalpy distribution along the vertical direction in the centerline of the regenerative oxidation bed for considering the influence of radiation or not at the conditions of $T_{\text {in }}=1200 \mathrm{~K}, q_{\text {in }}=0.00155 \mathrm{~kg} \cdot \mathrm{s}^{-1}$, $t=1600 \mathrm{~s}$. The enthalpy without the radiation heat transfer initially is higher than that with the radiation heat transfer. Then the difference between them decreases gradually along the vertical direction in the centerline of the regenerative oxidation bed. After $x=142 \mathrm{~mm}$, the enthalpy without the radiation heat transfer is smaller than that with the radiation heat transfer. This can be explained as follows. When the radiation is not care in the heating process, the heat transfer in the porous media just by conduction and convection, leading to less heat transfer along the vertical direction in the regenerative oxidation bed. So radiation takes an important role in the process of heat transfer, it is must be taken into account when the transient heat transfer in porous media is analyzed.

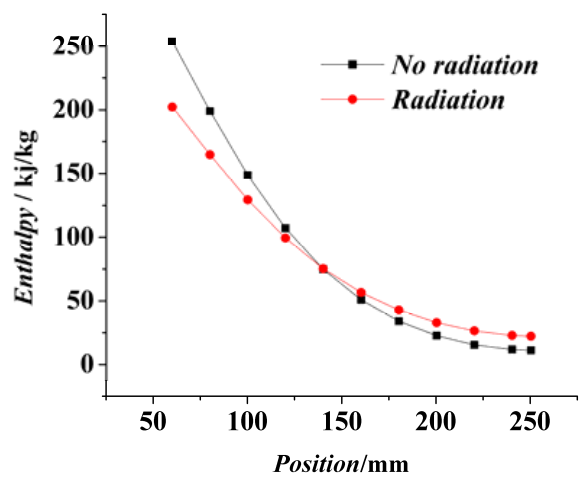

Fig. (10). The changes of enthalpy for considering the influence of radiation or not.

\subsection{The Temperature Distribution of the Regenerative Oxidation Bed}

Fig. (11) indicates the changes of temperature distribution of the regenerative oxidation bed along the direction of

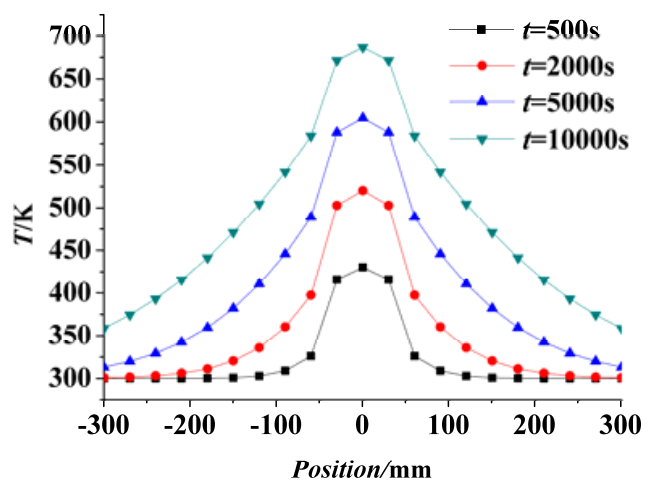

Fig. (11). The changes of temperature distribution of the regenerative oxidation bed (along the direction of height in the middle facet of it). 
height in the middle facet of it during the preheating process. It can be seen that the temperature is the highest in the middle of the regenerative oxidation bed, and it decreases gradually along the direction of height. With the preheating process progresses, the outlet temperature of the regenerative oxidation bed is increasing, which leads to the loss of more energy.

\section{CONCLUSIONS}

A numerical study is performed to explore the transient preheating process of the regenerative oxidation bed heated by the burned gas generated from a burner placed outside. The main conclusions are summarized as follows.

When the burned gas initially flows into the regenerative oxidation bed, the temperature of the gas is higher than the solid's. The difference of temperature becomes smaller gradually with the heat transfer going on. The hot gas passing through the manifold is the main resource of the heat to preheat the regenerative oxidation bed. Increasing the inlet mass flow of the pipe could shorten the time of preheating. As the main parameters of porous media, specific heat and porosity have a great influence on the heat storage capacity of the regenerative oxidation bed. The larger the specific heat is, the greater the heat storage capacity for the regenerative oxidation bed will be. While the porosity has an opposite effect on the heat storage capacity. The importance of solid phase radiation on the temperature profiles in porous media is numerically assessed, whose contribution on heat transfer in the preheating process can not be ignored. The temperature is the highest in the middle of the regenerative oxidation bed, and it decreases gradually along the direction of height of the regenerative oxidation bed.

\section{ACKNOWLEDGEMENT}

This study was financially supported by Key Program of National High Technology Research and Development Program of China (No. 2009AA063202).

\section{REFERENCES}

[1] G. Q. Niu, "Prospect and utilization status of Low concentration gas in vitiated air at mine", Ind. Safety Environ. Prot., vol. 28, pp. 3-5, 2002.
[2] Pablo Marin, Miguel A. G. Hevia, Salvador Ordonez and Fernando $\mathrm{V}$, "Combustion of methane lean mixture reverse flow reactors: comparison between packed and structured catalyst beds", Catalysis Today, vol. 105, pp. 701-708, 2005.

[3] S. Su, J. Agnew, "Catalytic combustion of coal mine ventilation air methane", Fuel, vol. 85, pp. 1201-1210, 2006.

[4] S. Su, A. Beath, H. Guo, and Cliff Mallettet, "Characteristics of coal mine ventilation air flows", J. Env. Manag., vol. 86, pp. 44-62, 2008.

[5] B. ZHeng, Y. Q. Liu, "Oxidation of coal mine ventilation air methane in thermal reverse-flow reactor", J. China Coal Soc., vol. 34, pp. 1475-1478, 2009.

[6] T. N. Haynes, C. Georgakis, and H. S. Caram, "The design of reverse flow reactors for catalytic combustion systems", Chem. Eng. Sci., vol. 50, pp. 401-416, 1995.

[7] Y. Q. Liu, R. X. Liu, ZH. Q. Gao, "Coal mine ventilation air methane thermal flow-reversal reactor", CHINA. Patent ZL2,008,102,498,60.3, December 27, 2008.

[8] T. W. Tong, and S. B. Sathe, "Heat transfer characteristics of porous radiant burners", Heat Transfer, vol. 113, pp. 423-428, 1991.

[9] I. Malico, and J. C. F. Pereira, "Numerical study on the influence of radiative properties in porous media combustion", J. Heat Transfer, vol. 123, pp. 951-957, 2001.

[10] P. Talukdar, S. C. Mishra, D. Trimis, and F. Durst, "Combined radiation and convection heat transfer in a porous channel bounded by isothermal parallel plates", Int. J. Heat Mass Transfer, vol. 47, pp. 1001-1013, 2004.

[11] Y. T. Yang, and M. L. Hwang, "Numerical simulation of turbulent fluid flow and heat transfer characteristics in heat exchangers fitted with porous media", Int. J. Heat Mass Transfer, vol. 52, pp. 29562965, 2009.

[12] K. Ichimiya, and T. Matsuda, "Effects of a porous medium on local heat transfer and fluid flow in a forced convection field", Heat Mass Transfer, vol. 40, pp. 1567-1576, 1997.

[13] C. Ben Kheder, B. Cherif and M. S. Sifaoui, "Numerical study of transient heat transfer in semitransparent porous medium", Renewable Energy, vol. 27, pp. 543-560, 2002.

[14] P. Talukdar, S. C. Mishraa, D. Trimisb, and F. Durst, "Combined radiation and convection heat transfer in a porous channel bounded by isothermal parallel plates", J. Quant. Spectrosc. Radiat. Transfer, vol. 47, pp. 1001-1013, 2004.

[15] M. Farzaneh, R. Ebrahimi, M. Shams, and M. Shafiey, "Twodimensional numerical simulation of combustion and heat transfer in porous burners", Eng. Lett., vol. 15, No. 2, EL 152 28, 2007.

[16] H. Amiri, S. H. Mansouri, and A. Safavinejad, "Combined conductive and radiative heat transfer in an anisotropic scattering participating medium with irregular geometries", Int. J. Therm. Sci., vol. 49, pp. 492-503, 2010.

[17] A. A. Mohamad, "Local analytical discrete ordinate method for the solution of the radiative transfer equation", Int. J. Heat Mass Transfer, vol. 39, pp. 1859-1864, 1996.

[18] P. X. Jiang, and X. C. Lu, "Numerical simulation and theoretical analysis of thermal boundary characteristics of convection heat transfer in porous media", Int. J. Heat Fluid Flow, vol. 28, pp. 1144-1156, 2007. 